\title{
Fundamental Relation between Electrical and Thermoelectric Transport Coefficients in the Quantum Hall Regime
}

\author{
B. Tieke, ${ }^{1}$ R. Fletcher, ${ }^{2}$ U. Zeitler, ${ }^{3}$ A. K. Geim, ${ }^{1}$ M. Henini,${ }^{3}$ and J.C. Maan ${ }^{1}$ \\ ${ }^{1}$ Research Institute for Materials, High Field Magnet Laboratory, University of Nijmegen, Toernooiveld, 6525 ED Nijmegen, \\ The Netherlands \\ ${ }^{2}$ Physics Department, Queen's University, Kingston, Ontario, Canada K7L 3N6 \\ ${ }^{3}$ Department of Physics, University of Nottingham, Nottingham NG7 2RD, United Kingdom
}

(Received 20 November 1996)

\begin{abstract}
The two components $S_{x x}$ and $S_{y x}$ of the phonon-drag thermoelectric power in two-dimensional electron gases (2DEGs) are found to be related by $S_{y x}=\alpha B\left(d S_{x x} / d B\right)$ in the integer and fractional quantum Hall regime. A similar relation exists for electrical resistivity, $\rho_{x x}=\alpha B\left(d \rho_{x y} / d B\right)$, and we show that experimentally the constant $\alpha$ is the same in both cases indicating the universal character of such relations for transport in 2DEGs. These results link the behavior of $S_{y x}$, which hitherto has not been understood, to that of $S_{x x}$ and thus opens a new way of explaining this quantity. [S0031-9007(97)03363-2]
\end{abstract}

PACS numbers: 73.40.Hm, 73.50.Jt, 73.50.Lw

More than 15 years after the discovery of the quantum Hall effect (QHE) [1], the experimentally observed relation by Chang and Tsui [2] between the two independent components of the resistivity tensor $\overleftrightarrow{\rho}$ of a two-dimensional electron gas (2DEG) in high magnetic fields $B$ (where $\left.\rho_{x y} \gg \rho_{x x}\right)$, i.e.,

$$
\rho_{x x}=\alpha_{r} B \frac{d \rho_{x y}}{d B},
$$

where $\alpha_{r}$ is a scaling constant, remains puzzling. The validity of Eq. (1) has been confirmed in a number of experimental studies [3-6] in both the integer and fractional QHE. It correctly describes not only the zeros in $\rho_{x x}$ when $\rho_{x y}$ is in a plateau, but also faithfully reproduces all the finer details of the magnetoresistance curve. The empirical relation has been studied theoretically by Vagner and Pepper [7] using a scaling argument, and more recently by Simon and Halperin [8] who proposed an analysis based on inhomogeneities. A phenomenological analysis of the peak heights of Shubnikov-de Haas oscillations by Coleridge et al. [9] suggested that $\alpha_{r}$ is related to the effects of small angle scattering in quantum transport. So far these studies have been limited to electrical transport, and it is therefore of interest to ask whether a similar relation also holds between tensor components of other transport phenomena; this paper answers the question in the affirmative for the case of the thermoelectric tensor.

The thermopower (TEP) tensor $\overleftrightarrow{\vec{S}}$, is defined by $\vec{E}=\overleftrightarrow{S} \nabla T$, where $\vec{E}$ is the electric field which appears in the sample as a result of an applied temperature gradient $\nabla T$, and in a perpendicular magnetic field has two independent components $S_{x x}$ and $S_{y x}$. The principal driving mechanisms for TEP are diffusion and phonon drag [10], but in this work we focus on drag since it dominates the experiments.

The phonon-drag component of $S_{x x}$ in 2DEGs is well understood both theoretically [11] and experimentally
[12-14], but in contrast $S_{y x}$ is hardly understood at all. The drag contribution to $S_{y x}$ is expected to be zero $[10,15]$, leaving only the diffusion contribution which, at high magnetic fields, is expected to behave like the derivative of $S_{x x}$ with respect to $B$. In three-dimensional degenerate semiconductors $S_{y x}$ indeed shows only a diffusion contribution and no phonon drag, even under conditions where phonon drag completely determines $S_{x x}$ [16]. However, experiments on 2DEGs reveal that $S_{y x}$ is dominated by phonon drag [12-14] with no diffusion contribution being evident. Further, its field dependence resembles the derivative of $S_{x x}$ which is only expected for diffusion. This unexpected behavior, which occurs in both the integer and fractional QHE (IQH and FQH) regimes, has remained a mystery for over a decade.

In this Letter we will show that, for a variety of $2 \mathrm{DEG}$ samples and a wide range of temperatures and fields, $S_{y x}$ is experimentally related to $S_{x x}$ by an equation analogous to Eq. (1),

$$
S_{y x}=\alpha_{s} B \frac{d S_{x x}}{d B} .
$$

Furthermore the factors $\alpha_{s}$ and $\alpha_{r}$ are the same for each sample, with very similar values between different samples. This is an important result for two reasons: (i) It expands the generality of Eq. (1) to another transport property. Instead of looking for an explanation of Eq. (1) specific to $\overleftrightarrow{\rho}$, it is now clear that one needs to explain a universal feature of transport in 2DEGs. (ii) It sheds a completely new light on the origin of phonon drag in $S_{y x}$ in 2DEGs and allows all previous experimental results to be integrated into a consistent framework.

In Fig. 1 experimental data are shown for $S_{y x}$ and $\rho_{x x}$, together with $S_{y x}^{\text {calc }}$ and $\rho_{x x}^{\text {calc }}$, in the IQH and $\mathrm{FQH}$ regime for a low-density, "high-mobility" sample $\left(n=1.75 \times 10^{15} \mathrm{~m}^{-1}, \mu=93.0 \mathrm{~m}^{2} / \mathrm{V} \mathrm{s}\right.$; for data on $S_{x x}$ see Ref. [17]). $\rho_{x x}^{\text {calc }}$ and $S_{y x}^{\text {calc }}$ are calculated curves 




FIG. 1. Example for the fractional QHE regime. (a) Longitudinal resistivity $\rho_{x x}$ and $\rho_{x x}^{\text {calc }}$ calculated with Eq. (1) at $T=297 \mathrm{mK}$, with $\alpha_{r}=0.030$. The curve for $\rho_{x x}^{\text {calc }}$ is offset by $2.5 \mathrm{k} \Omega$. (b) NE coefficient $S_{y x}$ and $S_{y x}^{\text {calc }}$ calculated from $S_{x x}$ with Eq. (2) for $T=504 \mathrm{mK}, \alpha_{s}=0.027$. The curve for $S_{y x}^{\text {calc }}$ is offset by $0.5 \mathrm{mV} / \mathrm{K}$. Special filling factors $\nu$ are indicated in the graph. The inset in the upper panel shows the temperature dependence for fit parameters $\alpha_{r}(\triangle)$ and $\alpha_{s}$ (A) obtained in the range $B=1-20 \mathrm{~T}$. Both are constant and agree within experimental error.

obtained from the measured $\rho_{x y}$ and $S_{x x}$ using Eqs. (1) and (2) and numerically evaluated derivatives $d \rho_{x y} / d B$ and $d S_{x x} / d B$, with $\alpha_{r}$ or $\alpha_{s}$ as a free parameter to give the best fit to the $\rho_{x x}$ and $S_{y x}$ experimental data. We define a quality factor $\Delta=\int\left(S_{y x}-S_{y x}^{\text {calc }}\right)^{2} d B / \int\left[S_{y x}^{2}+\right.$ $\left.\left(S_{y x}^{\text {calc }}\right)^{2}\right] d B$ to quantify the relative deviation between the two curves; $\Delta=0$ for perfect agreement and $\Delta=1$ if no correlation exists. The agreement between $S_{y x}$ and $S_{y x}^{\text {calc }}$ is remarkable $(\Delta=0.09)$, reproducing the position and shape of the oscillations with their characteristic sign changes, as well as the relative magnitudes in the IQH and FQH regimes almost perfectly. A slight discrepancy can be seen around the even denominator filling factors $\nu=\frac{1}{2}, \frac{3}{2}, \frac{1}{4}$, and $\frac{3}{4}$ where $S_{y x}^{\text {calc }}$ vanishes, whereas the experimental $S_{y x}$ is finite. However, this additional background in the fractional QHE, which is clearly of a different origin, does not significantly detract from the overall agreement between $S_{y x}^{\text {calc }}$ and $S_{y x}$. In fact, the value of $\alpha_{s}$ we obtain is insensitive to whether this background is subtracted or not. The inset to Fig. 1 shows that $\alpha_{s}$ and $\alpha_{r}$ are independent of temperature. This is consistent with previous observations that $\alpha_{r}$ is constant for $T \lesssim 40 \mathrm{~K}[3,18]$, but a constant $\alpha_{s}$ for TEP is more noteworthy since, in contrast to $\rho_{x y}$, the absolute magnitude of both $S_{x x}$ and $S_{y x}$ varies by several orders of magnitude in this temperature range due to phonon drag [12-14,17]. This result also stresses the common origin of $S_{x x}$ and $S_{y x}$.

To test the validity of Eq. (2) over a wider parameter range, we have analyzed a variety of GaAs $/ \mathrm{Ga}_{1-x} \mathrm{Al}_{x}$ As heterostructures, including previously published data [12-14]. Together with the new data, the analysis covers broad ranges of temperature $(T=0.2-10 \mathrm{~K})$, density $\left[n=(0.4-6.7) \times 10^{15} \mathrm{~m}^{-2}\right]$, mobility $\left(\mu=3.8-266 \mathrm{~m}^{2} / \mathrm{V} \mathrm{s}\right)$, and magnetic field $(B=1-30 \mathrm{~T})$, and both the integer and fractional QHE regime. Figure 2 gives a typical example for the measured quantities, $\rho_{x x}$ and $S_{y x}$, and calculated curves, $\rho_{x x}^{\text {calc }}$ and $S_{y x}^{\text {calc }}$, for a sample with a higher density and a lower mobility than the previous one (A4 in Ref. [13]), highlighting the behavior in the integer QHE regime. Again $S_{y x}^{\text {calc }}$ strikingly resembles $S_{y x}$, with a quality $(\Delta=0.05)$ similar to that for $\overleftrightarrow{\rho}(\Delta=0.08)$. The only obvious discrepancy between $S_{y x}^{\text {calc }}$ and $S_{y x}$ is that the derivative tends to accentuate structure which is weak in the experimental $S_{y x}$ as can be seen for the developing spin splitting at $\nu=5$ in Fig. 2. This behavior is also found for $\rho_{x x}^{\text {calc }}$

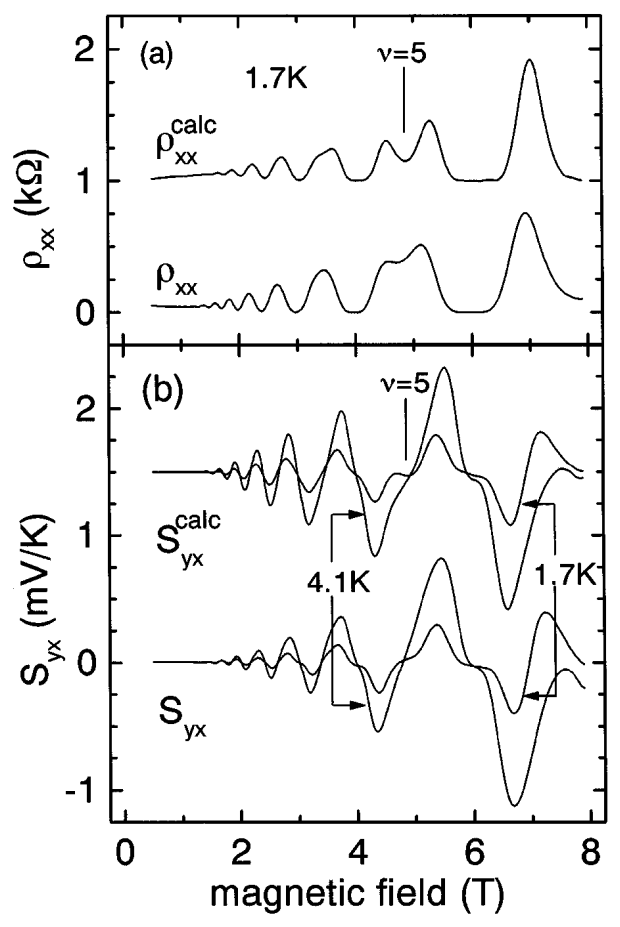

FIG. 2. Example for the integer QHE regime. (a) Longitudinal resistivity $\rho_{x x}$ and $\rho_{x x}^{\text {calc }}$ calculated from the Hall resistance $\rho_{x y}$ with Eq. (1), $\alpha_{r}=0.033$. The curve for $\rho_{x x}^{\text {calc }}$ is offset by $1 \mathrm{k} \Omega$. (b) NE coefficient $S_{y x}$ and $S_{y x}^{\text {calc }}$ calculated from $S_{x x}$ with Eq. (2) for $T=4.12 \mathrm{~K}(1.74 \mathrm{~K})$, with $\alpha_{s}=0.027(0.022)$. The curves for $S_{y x}^{\text {calc }}$ are offset by $1.5 \mathrm{mV} / \mathrm{K}$. Original experimental data are taken from Ref. [13], sample A4. 
[6] and can be seen in Fig. 2. Summarizing, the fit of Eqs. (1) and (2) to all investigated data is very good with a quality factor $\Delta$ in all cases better than 0.1 , and often less than 0.03 , for a wide range of densities, temperatures, and fields.

Another striking result is that the required values of $\alpha_{s}$ and $\alpha_{r}$ are always very similar as is obvious from the figures, especially the inset to Fig. 1. Figure 3 summarizes the results for $\alpha_{s}$ and $\alpha_{r}$ for many different samples with different mobilities $\mu$ and densities $n$. The values lay in the range $0.01-0.07$ for all samples, showing no clear dependence on $\mu$ or $n$ though they tend to decrease for higher mobilities, a trend which is most pronounced if $\mu$ is increased by persistent photoconductivity. The standard deviation of the fits for $\alpha_{s}$ and $\alpha_{r}$ is $\sim 15 \%$. Systematic uncertainties may arise with TEP from the dimensions of the sample, which, unlike the resistivity, are reflected directly in $\alpha_{s}$. These errors are sample specific and are typically $\sim 20 \%$. Taking all samples [19] into account, we see no significant difference between $\alpha_{s}$ and $\alpha_{r}$ and conclude that the two scaling parameters are identical within experimental error.

More insight in the emperical relation Eq. (2) can be gained by inspection of the tensor relations between the electric current density $\vec{J}$ produced by an applied electric field $\vec{E}$, and the thermal current density $\vec{U}$ due to a temperature gradient $\nabla T$

$$
\begin{aligned}
\vec{J} & =\overleftrightarrow{\sigma} \vec{E}-\overleftrightarrow{\epsilon} \nabla T, \\
\vec{U} & =T \overleftrightarrow{\epsilon} \vec{E}-\overleftrightarrow{\lambda} \nabla T,
\end{aligned}
$$


FIG. 3. Scaling parameters $\alpha_{r}$ (open symbols) and $\alpha_{s}$ (closed symbols) as a function of (a) mobility and (b) density for a variety of samples. Data points are connected for the same sample illuminated (marked by arrows) and nonilluminated. Original data are taken from Ref. [12]; samples 2 and $3(\bullet)$,

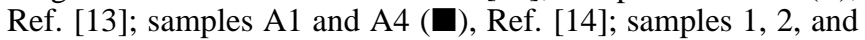
$3(\boldsymbol{\nabla})$; and samples from the same wafer as the sample in Fig. 1 (A). with $\overleftrightarrow{\sigma}$ the electrical conductivity, $\overleftrightarrow{\epsilon}$ the thermoelectric tensor, and $\overleftrightarrow{\lambda}$ the thermal conductivity. Because of the Onsager symmetry relations, $\overleftrightarrow{\epsilon}$ links Eq. (3a) to (3b) giving rise to an electric current due to $\nabla T$ in Eq. (3a) and a heat current due to $\vec{E}$ in Eq. (3b). In a TEP experiment there is no net current $(\vec{J}=0)$ and the TEP tensor defined above is given by $\overleftrightarrow{S}=\overleftrightarrow{\rho} \overleftrightarrow{\epsilon}$.

The similarity between Eqs. (1) and (2) could arise because $\overleftrightarrow{\epsilon}$ and $\overleftrightarrow{\sigma}$ fulfill similar roles in Eqs. (3), each relating a driving force $(\vec{E}$ or $\nabla T)$ to a current density $(\vec{J}$ or $\vec{U})$. Therefore, because at high magnetic fields Eq. (1) is equivalent to $\sigma_{x x}=\alpha_{r} B\left(d \sigma_{x y} / d B\right)$ [5], it is reasonable to assume that the two independent components of $\overleftrightarrow{\epsilon}, \epsilon_{x x}$ and $\epsilon_{x y}$, are related in a similar way, namely, $\epsilon_{x x}=\alpha_{s} B\left(d \epsilon_{x y} / d B\right)$, where $\alpha_{s}$ is not a priori identical to $\alpha_{r}$. Using $\nabla T_{y}=0$ (i.e., no transverse temperature gradient) gives $S_{x x}=\rho_{x x} \epsilon_{x x}+\rho_{y x} \epsilon_{x y}$ and, omitting the term $\rho_{x x} \epsilon_{x x}$ which is experimentally found to be negligible, it follows that

$$
\begin{aligned}
\alpha_{s} B \frac{d S_{x x}}{d B} & =\alpha_{s} B \frac{d \epsilon_{x y}}{d B} \rho_{y x}+\alpha_{s} B \frac{d \rho_{y x}}{d B} \epsilon_{x y} \\
& =\rho_{y x} \epsilon_{x x}+\rho_{x x} \epsilon_{y x}+\frac{\Delta \alpha}{\alpha_{r}} \rho_{x x} \epsilon_{y x}
\end{aligned}
$$

where $\Delta \alpha=\alpha_{s}-\alpha_{r} \simeq 0$. Since $\rho_{x x} \epsilon_{y x}$ is at most $10 \%$ of $\rho_{y x} \epsilon_{x x}$ the last term becomes completely negligible and Eq. (2) follows immediately from Eq. (4) since $S_{y x}=$ $\rho_{x x} \epsilon_{y x}+\rho_{y x} \epsilon_{x x}$.

Since $\alpha_{s}$ and $\alpha_{r}$ are practically identical over a wide range of experimental parameters, the physical mechanism responsible for the effect must be common to both $\overleftrightarrow{\rho}$ and $\overleftrightarrow{S}$. The available evidence indicates that the relationships of Eqs. (1) and (2) are specific to the case of 2DEGs and are not exhibited by 3D degenerate gases. As noted above, it has been suggested [9] that $\alpha_{r}$ is related to small angle scattering in magnetotransport through its different effects on Landau level broadening and the momentum relaxation time. Such a mechanism seems hard to invoke for phonon drag TEP which primarily reflects the strongly temperature dependent electron-phonon scattering rate [20].

A hint to a plausible explanation is provided by a recent theory by Simon and Halperin [8] who suggested that Eq. (1) can be explained by macroscopic concentration fluctuations in the 2DEG. They argue that in resistivity the macroscopically measured $\rho_{x x}$ is mainly governed by fluctuations in $\rho_{x y}$ and only weakly dependent on the local value of $\rho_{x x}$. Extending this argument to TEP, the macroscopically measured Nernst-Ettingshausen coefficient $S_{y x}$ will mainly depend on fluctuations in $S_{x x}$ and not on the local value of $S_{y x}$ (which is expected to vanish for phonon drag). Therefore concentration fluctuations would have a similar relative effect on $S_{x x}$ and $\rho_{x y}$ implying that the scaling behavior for resistivity and TEP, as manifested in 
Eqs. (1) and (2) with $\alpha_{r} \approx \alpha_{s}$, is the same. The experimental fact that $\alpha$ tends to decrease upon illumination of the sample, which is known to increase the homogeneity, is consistent with this theory. On the other hand, the value of $\alpha$ experimentally obtained would imply density fluctuations of the order of 5\%, which appears unreasonably large, as mentioned by the authors [8].

In summary, we have shown that the empirical relation Eq. (1) between the tensor components for electrical transport in quantum Hall systems has an analog Eq. (2) valid for thermoelectric transport and that the dimensionless scaling factors are identical. This result shows that such relations have a much more general validity than to resistivity only, and at the same time links observed phonon-drag Nernst-Ettingshausen coefficient $S_{y x}$ to the behavior of $S_{x x}$ in a new manner, thus opening the way to a totally different explanation than the usual, as yet unsuccessful, theoretical approaches. On the basis of these results it might be speculated that relations similar to Eqs. (1) and (2) will exist for other properties of a 2DEG, e.g., diffusion TEP, acoustoelectric effect, and electronic thermal conductivity.

This work has been supported by the European Commission under Contract No. CHGE-CT93-0051 (Large Installation Plan) and, in part, by NSERC, Canada.

[1] K. von Klitzing, G. Dorda, and M. Pepper, Phys. Rev. Lett. 45, 494 (1980).

[2] A. M. Chang and D. C. Tsui, Solid State Commun. 56, 153 (1985).

[3] T. Rötger, G. J.C.L. Bruls, J.C. Maan, P. Wyder, K. Ploog, and G. Weimann, Phys. Rev. Lett. 62, 90 (1989).

[4] N. G. Morawicz, K. W. J. Barnham, C. Zammit, J. J. Harris, C. T. Foxon, and P. Kujawinski, Phys. Rev. B 41, 12687 (1990).

[5] H.L. Stormer, K.W. Baldwin, L. N. Pfeiffer, and K.W. West, Solid State Commun. 84, 95 (1992).
[6] A. A. Allerman, W. Xu, N. Hauser, and C. Jagadish, J. Appl. Phys. 77, 2052 (1995).

[7] I. D. Vagner and M. Pepper, Phys. Rev. B 37, 7147 (1988).

[8] S. H. Simon and B. I. Halperin, Phys. Rev. Lett. 73, 3278 (1994).

[9] P. T. Coleridge, P. Zawadzki, and A. S. Sachrajda, Phys. Rev. B 49, 10798 (1994).

[10] B.L. Gallagher and P. N. Butcher, in Handbook on Semiconductors, edited by P.T. Landsberg (Elsevier, Amsterdam, 1992), Vol. 1, pp. 721-816.

[11] S. S. Kubakaddi and P. N. Butcher, Phys. Rev. B 40, 1377 (1989).

[12] R. Fletcher, J. C. Maan, K. Ploog, and G. Weimann, Phys. Rev. B 33, 7122 (1986).

[13] R. Fletcher, M. D'Iorio, W. T. Moore, and R. Stoner, J. Phys. C 21, 2681 (1988).

[14] U. Zeitler, J. C. Maan, P. Wyder, R. Fletcher, C. T. Foxon, and J. J. Harris, Phys. Rev. B 47, 16008 (1993).

[15] T. M. Fromhold, P. N. Butcher, G. Qin, B. G. Mulimani, J. P. Oxley, and B.L. Gallagher, Phys. Rev. B 48, 5326 (1993).

[16] B. Tieke, R. Fletcher, J.C. Maan, W. Dobrowolski, A. Mycielski, and A. Wittlin, Phys. Rev. B 54, 10565 (1996).

[17] B. Tieke, U. Zeitler, R. Fletcher, S. A. J. Wiegers, A. K. Geim, J.C. Maan, and M. Henini, Phys. Rev. Lett. 76, 3630 (1996).

[18] D. R. Leadley, R. J. Nicholas, W. Xu, F. M. Peeters, J. T. Devreese, J. Singleton, J. A. A. J. Perenboom, L. van Bockstal, F. Herlach, C. T. Foxon, and J. J. Harris, Phys. Rev. B 48, 5457 (1993).

[19] For one low-density, low-mobility sample (No. 4 from Ref. [12]) we find an enhanced $\alpha_{r}$ of about $2 \times \alpha_{s}$. We attribute this to the sample being close to a Hallinsulator state. We do not expect Eq. (1) to be a good description for such states as is obvious from the strongly temperature dependent $\rho_{x x}$ in the insulating phase for filling factors close to $\nu=\frac{1}{5}$ which is observed in highmobility 2DEGs.

[20] W. Kang, Song He, H. L. Stormer, L. N. Pfeiffer, W. Baldwin, and K. W. West, Phys. Rev. Lett. 75, 4106 (1995). 